\title{
COLLISION-FREE TRAJECTORY PLANNING FOR MANIPULATORS USING GENERALIZED PATTERN SEARCH
}

\author{
Ata, A. A. \& Myo, T. R. \\ Mechatronics Engineering Department, Faculty of Engineering \\ International Islamic University Malaysia, P.O. Box 10, Kuala Lumpur 50728, Malaysia \\ E-Mail: atef@iiu.edu.my
}

\begin{abstract}
A Generalized Pattern Search algorithm (GPS) is introduced to design a collision-free trajectory for planar redundant manipulators. Cubic-spline interpolation trajectory for the endeffector is designed a priori by selecting three intermediate points around the workspace obstacles. The GPS algorithm ensures that none of the selected points lies inside any of the obstacles. The end-effector will follow the prescribed trajectory in excellent manner without colliding with the obstacles. Simulation studies are carried out for three different obstacles to prove the validity of the proposed algorithm.

(Received in April 2006, accepted in October 2006. This paper was with the authors 1 month for 1 revision.)
\end{abstract}

Key Words: Redundant Manipulator, Genetic Algorithms, Generalized Pattern Search, Obstacle Avoidance, Cubic-Spline, Optimization

\section{INTRODUCTION}

In many today's manufacturing applications such as welding, spray painting, a robot manipulator must traverse with its end-effector a desired trajectory, while its body avoids collisions with the obstacles in the environment. The obstacle avoidance problem where robotic manipulators are required to move from an initial position to a specified final goal without colliding with any obstacles in the workspace is very important. Obstacle avoidance considering full manipulator dynamics is pretty difficult because of non-linearity of manipulator dynamics and existence of obstacles. An alternative approach is to use some optimization techniques rather than the inverse jacobian to save computation time and not to be trapped by singular points. In the last two decades, Genetic Algorithms (GAs) have been successfully applied to solve the obstacle avoidance trajectory planning problem of robot manipulators. Genetic Algorithms (GAs) are robust optimization tools based on natural evolution for complex search problems which can cope with discontinuities, non-linearities and even noisy [3].

A series of researchers applied the GAs technique to solve the collision-free trajectory planning of manipulators. Shiller and Dubowsky [12] proposed a method to solve optimal trajectory with collision-free problem. A small number of candidates of optimal trajectory in a discretized workspace were searched for. Then the trajectory is improved using the gradient method. It is easy predicted that it takes too much time all over the workspace. Yamamoto et al. [16] presented a method for time optimal collision-free trajectory planning based on both the Genetic Algorithms and the gradient method using an iteratively improving approach based on a gradient method. Their approach applies two global methods, a Genetic Algorithms to search multiple initial feasible spatial paths for the gradient method. Next, the gradient method searches time optimal solution locally within the multiple initial feasible solutions. The spatial path is represented only by the control points of B-spline. Therefore, if a boundary conditions and dynamical constraints of the problem are specified, the minimum 
traveling time can be estimated by only the control points. Rana and Zalzala [11] developed a method to plan a near time-optimal, collision-free motion in the case of multi-arm manipulators. The planning is carried out in the joint space and the path is represented as a string of via points connected through cubic spline. Doyle and Jones [2] proposed a pathplanning scheme that uses a GAs to search the manipulator configuration space for optimum path. The GAs generate good path solutions but it is not sufficiently robust. Lee and Lee [4] proposed a genetic trajectory planning of a robot manipulator producing the optimum trajectory between two points. They investigated the proper genetic trajectory parameterization and developed an efficient scheme for the implementation of genetic trajectory planner. Pires et al. [10] presented a method based on GAs adopting the direct kinematics. The optimal manipulator is the one that minimizes both the path trajectory length and the ripple in the time evolution without any collision with the obstacles in the workspace. Simulation results involving different robot structures and trajectories in the workspace were carried out to validate the proposed approach.

Nearchou and Aspragathos applied various heuristic techniques to solve the obstacle avoidance problem based on known notions and structures from computational geometry and computer graphics $[6,7,8]$. A new technique based on the concept of convex-hulls was proposed [6]. This technique guarantees detection of an imminent collision between a robot's link and an obstacle by constructing the convex-hull of the following points. An algorithm for continuous motion on a desired trajectory which computes in each step of the robot movement a small change in the vector of the robot's joints displacement was presented [7]. These movements should satisfy three criteria: the direction of the motion, the proximity to the desired curve, and the obstacle avoidance. In 1998, they continued by presenting an algorithm for trajectory generation under maximum allowed deviation [8]. The algorithm combines Raster techniques and GAs so that the tip traverses a trajectory within a maximum allowed position deviation from the desired curve. Pack et al., [9] proposed a Genetic Algorithms based method to search for feasible trajectory to avoid point obstacles in the configuration space. Their approach can also be extended to n-dimensional space. Tian and Collins [13] proposed a Genetic Algorithms using a floating point representation to search for optimal end-effector trajectory for a redundant manipulator. An evaluation function based on multiple criteria such as total displacement of all joints and the uniformity of Cartesian and joint space velocities was introduced. To verify their approach, simulations are carried out in free space and in a workspace with obstacles. While in 2004, they extended their work by developing a novel Genetic Algorithms for point obstacles avoidance trajectory using a cubic interpolation function [14]. Their algorithm searches several interior points between the starting and target points and uses the Hermit cubic interpolation to construct the path. Merchan-Cruz and Morris [5] extended the application of GAs for a collision-free of a system of two manipulators using potential field approach. In this approach each manipulators is considered as a moving obstacle by the other and collision is avoided. The GAs carry parallel optimization to find the best configuration for collision-free as well as minimizing the error to their respective goals.

In this work, a new hybrid optimization algorithm combining Genetic Algorithms and direct search as a Generalized Pattern Search (GPS) is utilized to design an obstacle avoidance trajectory for redundant manipulators. This algorithm possesses excellent tracking performance when examined to design optimal trajectory for redundant manipulators [1]. A three intermediate points are selected around the object and collision-free trajectory will be designed based on cubic-spline function. The end-effector will track the designed trajectory without colliding with the obstacle. Three case studies are simulated using MATLAB to validate the proposed algorithm. 


\section{PROBLEM FORMULATION}

Consider the three degree-of-freedom planar robotic arm shown in Fig. (1) where the endeffector is required to move from starting point to goal point without colliding with the workspace obstacles. In order to design the optimized joints trajectory accurately a complete description of the environment must be known in advance (e.g. shape and orientation of the obstacles). In this study, the robot joints are assumed to rotate 360 degree without any physical limits.

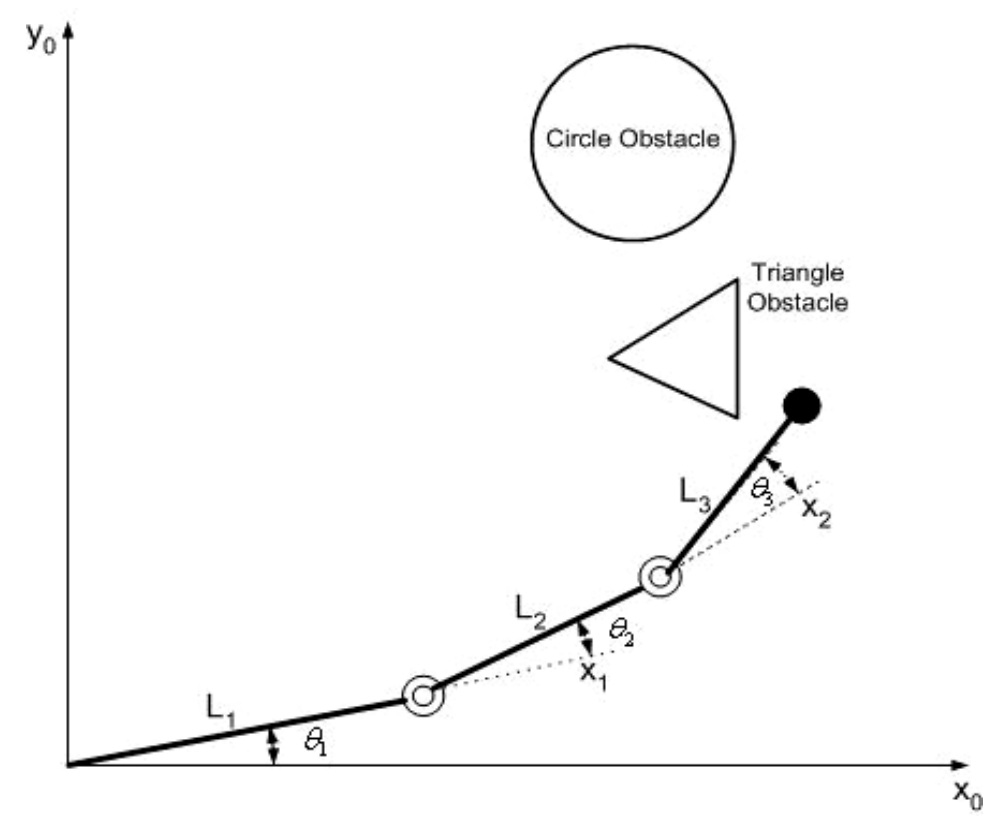

Figure 1: Three d-o-f planar robot configuration.

The main target here is to search for the optimized joints angles so the end-effector can reach the target without collision with the obstacles.

\section{GENERALIZED PATTERN SEARCH (GPS)}

Actually, there are two main advantages for Genetic Algorithms approach to solve the obstacle avoidance problem of manipulators. First, Genetic Algorithms based methods seldom require a priori knowledge of the problem. Furthermore, they do not fall into local optima and proceed toward global optima. However, they have difficulty in handling equality constraints of trajectory boundary conditions because they use probabilistic transition rules to find a solution [10,16]. Also there are some drawbacks of GAs in terms of inconsistency of the solution even if one started from the same population and it is also time consuming. To solve some of these drawbacks, Wetter and Wright [15] suggested that combining Genetic Algorithms with any method of coordinated search technique will improve the behavior of the system. Ata and Myo [1] also proved that using a Generalized Pattern Search (GPS) which consists of Genetic Algorithms as well as Direct Search gives excellent results in terms of trajectory tracking with minimum tracking error.

The Generalized Pattern Search is a hybrid optimization algorithm combining Genetic Algorithm used for the global search and Pattern Search Algorithm for the local search. The Genetic Algorithms have the advantage not to be trapped in local optima and proceeds toward global optima because GAs search from a population not a single point. Adding the Pattern 
Search optimization method refines the obtained result at each stage.

As with any optimization routine, the evaluation function provides the mechanism for determining the direction of the search. The evaluation or fitness function is defined based on end-effector positioning error and joint angle displacements satisfying Cartesian and joint velocity uniformity. Since a hybrid optimization approach is applied, the evaluation function is defined separately for Genetic Algorithms and Pattern Search methods. The objective function for GAs is defined as follows:

$$
F_{o b j}=C_{1} E_{e}+C_{2} D_{j}
$$

where, $\mathrm{E}_{\mathrm{e}}$ is the error between desired position and generated position of end-effector, $D_{j}$ is the joint displacements between successive points and $C_{1}$ and $C_{2}$ are weighting factors to control the desired configuration which satisfy the constraint $C_{1}+C_{2}=1$. Since the objective is to minimize the error between the desired and generated position of end-effector, $E_{e}$ will be defined as:

$$
E_{e}=\sum_{i=1}^{n} \sqrt{\left(x^{i}-x_{g}^{i}\right)^{2}+\left(y^{i}-y_{g}^{i}\right)^{2}}
$$

where, $\left(x^{i}, y^{i}\right)$ are desired end-effector positions and $\left(x_{g}^{i}, y_{g}^{i}\right)$ are generated end-effector positions. The joint displacements between successive points are considered in evaluation function in order to minimize actuator motions. To minimize the joint movements along the trajectory, the function will be

$$
D_{j}=\sum_{i=1, k=3}^{i=n, k=3}\left(\theta_{k}^{i+1}-\theta_{k}^{i}\right)^{2}
$$

where $\theta_{k}^{i}$ is the current joint angle for joint number $\mathrm{k}$ and $\theta_{k}^{i+1}$ is the next joint angle for joint number $\mathrm{k}, \mathrm{k}$ is the link number of robot manipulator and $\mathrm{n}$ is the number of knot points in trajectory. Pattern Search can be only used to reduce the tracking error of end-effector, so the evaluation function will be defined as.

$$
F_{\text {eval }}=\sum_{i=1}^{n} \sqrt{\left(x^{i}-x_{g}^{i}\right)^{2}+\left(y^{i}-y_{g}^{i}\right)^{2}}
$$

\section{SIMULATION AND DISCUSSION}

In order to validate the proposed algorithm, the end-effector trajectory is designed to move from starting to goal points avoiding obstacles in the workspace. Two approaches are considered here. The first one describes the trajectory generation when all the obstacles coordinates are known a priori. A cubic-spline interpolation function is used to provide a smoother trajectory based on three selected intermediate points between the start and goal at each generation. These three intermediate points will be chosen outside the obstacles in the course of motion.The proposed Generalized Pattern Search algorithm will optimize the joint angles so the end-effector tracks the prescribed trajectory smoothly and accurately without colliding with any obstacles. This type of trajectory can be applied mainly for off-line applications.

Three cases are considered here and the simulations are carried out using MATLAB toolbox of Genetic Algorithm and Direct Search. In the first case, a single circle is placed as an obstacle with radius $0.15 \mathrm{~m}$ and center located at $(0.4 \mathrm{~m}, 0.7 \mathrm{~m})$. The starting and target 
points are $(0.8,0.4) \mathrm{m}$ and $(0.1,0.9) \mathrm{m}$ respectively and the initial joint configurations are considered as $\theta_{1}^{1}=-10^{\circ}, \theta_{2}^{1}=60^{\circ}$ and $\theta_{3}^{1}=-30^{\circ}$. In the second case, two circles are placed in workspace as obstacles. The larger circle is centered at $(0.4,0.7) \mathrm{m}$ with radius $0.15 \mathrm{~m}$ while the smaller circle is centered at $(0.5,0.4) \mathrm{m}$ with radius $0.1 \mathrm{~m}$. The starting and target points are defined as $(0.6,0.25) \mathrm{m}$ and $(0.25,0.9) \mathrm{m}$ respectively with initial joint configurations considered as $\theta_{1}^{1}=-20^{\circ}, \theta_{2}^{1}=160^{\circ}$ and $\theta_{3}^{1}=-65^{\circ}$. While in the third case, a circle and a triangle are placed in workspace as obstacles. The circle specification is the same as the larger circle of second case. The corner points of triangle are located at $(0.4,0.4) \mathrm{m}$, $(0.6,0.3) \mathrm{m}$ and $(0.6,0.5) \mathrm{m}$ respectively. The starting and target points are defined as $(0.25$, $0.9) \mathrm{m}$ and $(0.6,0.25) \mathrm{m}$. The initial joint configurations are considered as $\theta_{1}^{1}=130^{\circ}$, $\theta_{2}^{1}=-35^{\circ}$ and $\theta_{3}^{1}=0^{\circ}$.

In case of a triangular obstacle, it can be approximated as a circle passing through its three vertices where its center is the geometric center of the triangle. This adds more flexibility of the proposed algorithms to handle more obstacles of complex geometric shapes. In all the three cases considered here, three points are selected between starting point and target point and are then interpolated by using cubic-spline. After ensuring trajectory is not colliding with obstacles, Generalized Pattern Search (GPS) is used to search for optimized joint angles to follow resulted trajectory minimizing both displacement error and joint angles movements. The parameters applied for simulation are summarized in Tables I and II.

Table I: Genetic Algorithm parameters.

\begin{tabular}{|l|l|}
\hline Population & 100 \\
\hline Fitness scaling & Rank \\
\hline Selection & Stochastic Uniform \\
\hline Reproduction & Elite count $=2$, crossover rate $=1.2$ \\
\hline Mutation & Gaussian(scale $=1$, shrink $=1$ ) \\
\hline crossover & Scattered function \\
\hline Migration & Forward (fraction 0.2, Interval $=20)$ \\
\hline Generation & 200 \\
\hline
\end{tabular}

Table II: Pattern Search parameters.

\begin{tabular}{|l|l|}
\hline Poll & Complete \\
\hline Search & Complete \\
\hline Polling Order & Consecutive \\
\hline
\end{tabular}

The simulated results for the three cases are shown in Fig. 2, 3, and 4. Fig. 2a, 3a, and 4a represent the robot configurations while Fig. $2 b$, $3 b$, and $4 b$ show the optimized joint angles. 

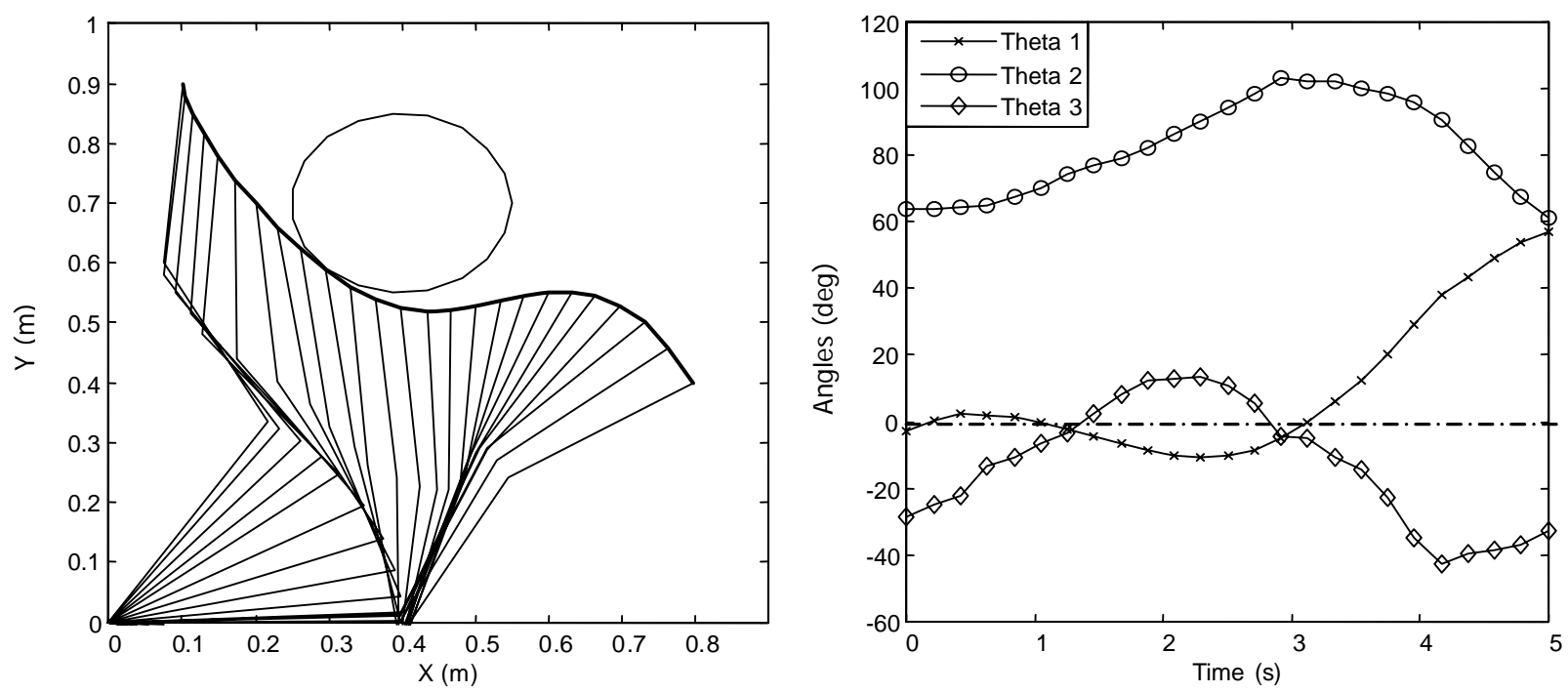

Figure 2a: Robot configuration with one circle.

Figure 2b: Optimized joint angles.
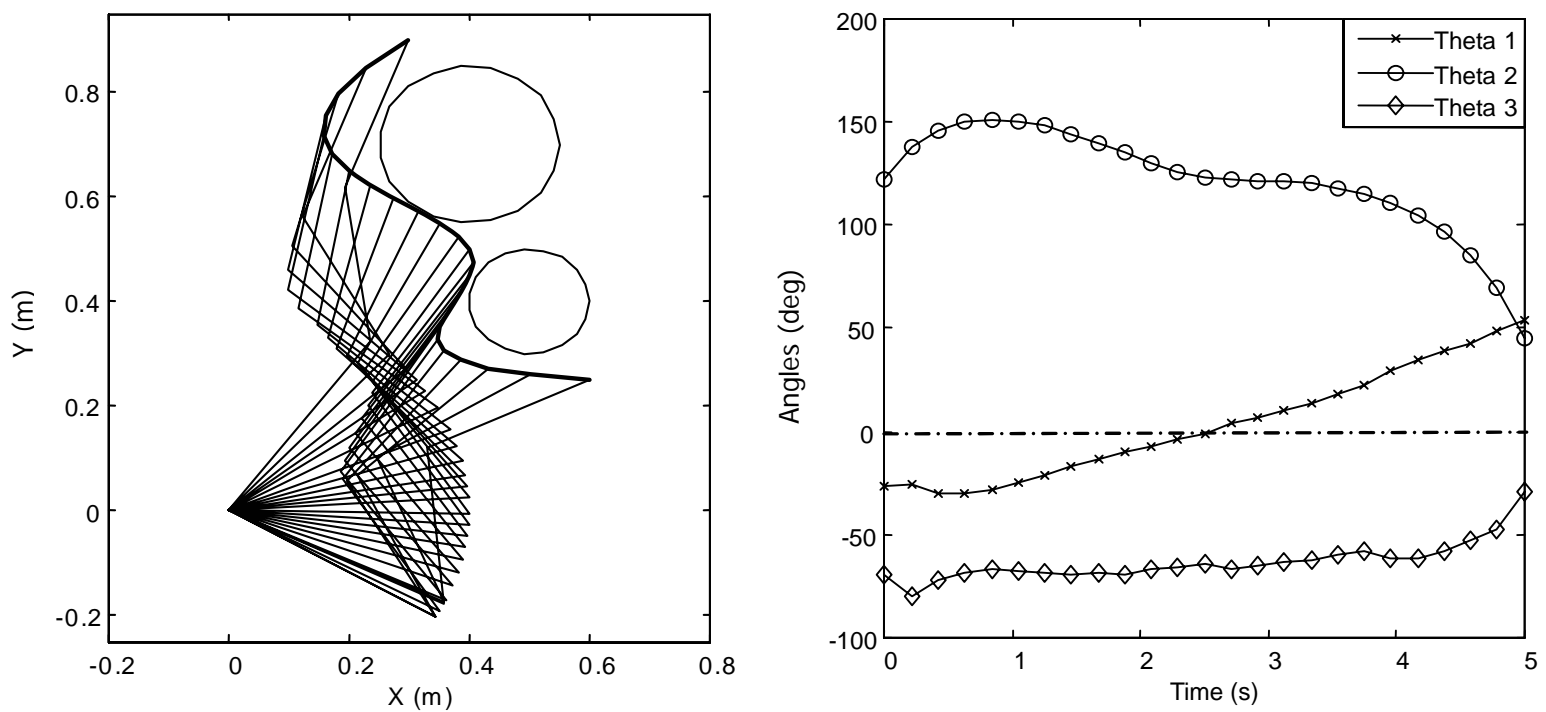

Figure 3a: Robot configuration - two circles.
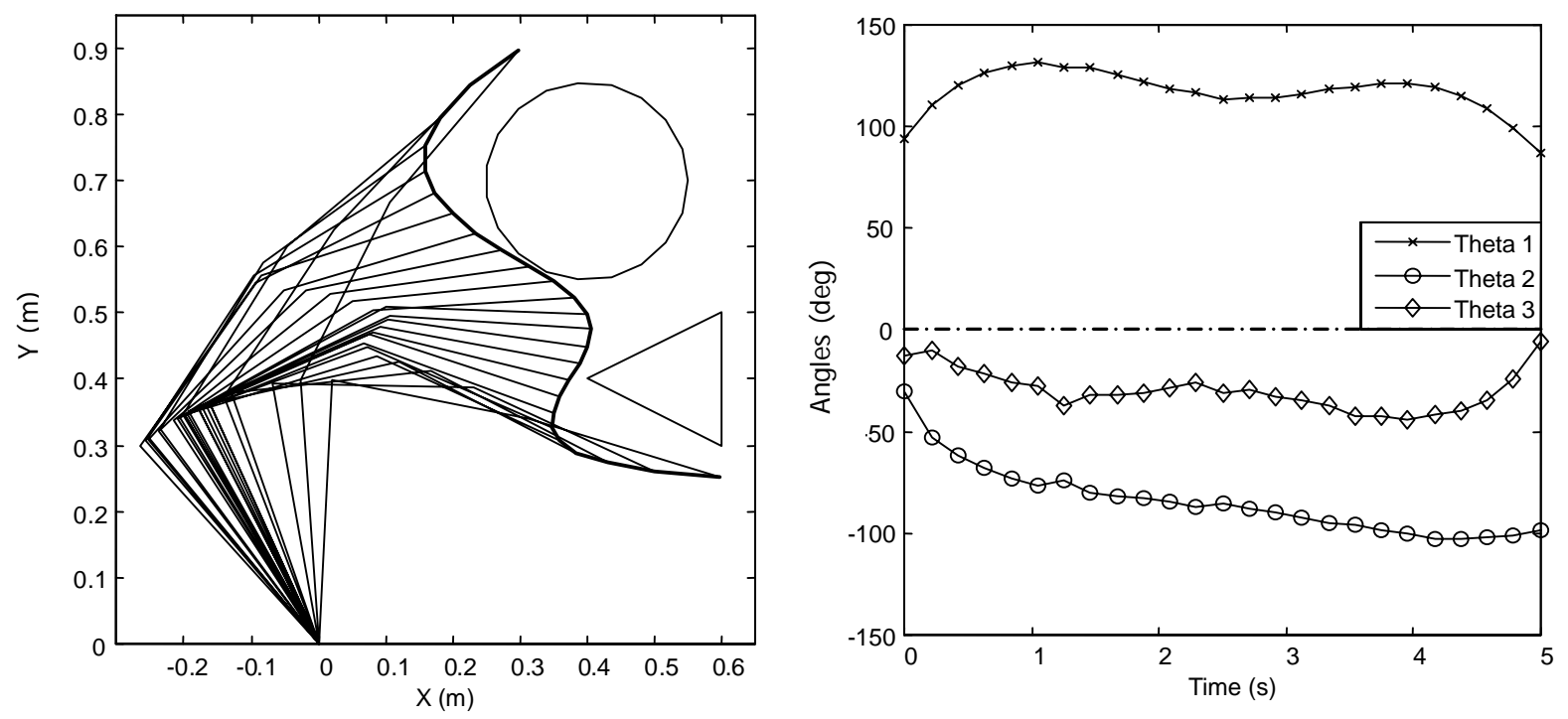

Figure 4a: Robot configuration - circle and triangle. $\quad$ Figure 4b: Optimized joint angles. 
The second approach can be used for online obstacle avoidance trajectory planning. The flowchart indicating the Generalized Pattern Search Algorithm for the collision avoidance trajectory is shown in Fig. 5 where the desired trajectory for manipulators, with end-effectors moving from a specified starting point to the goal point is generated.

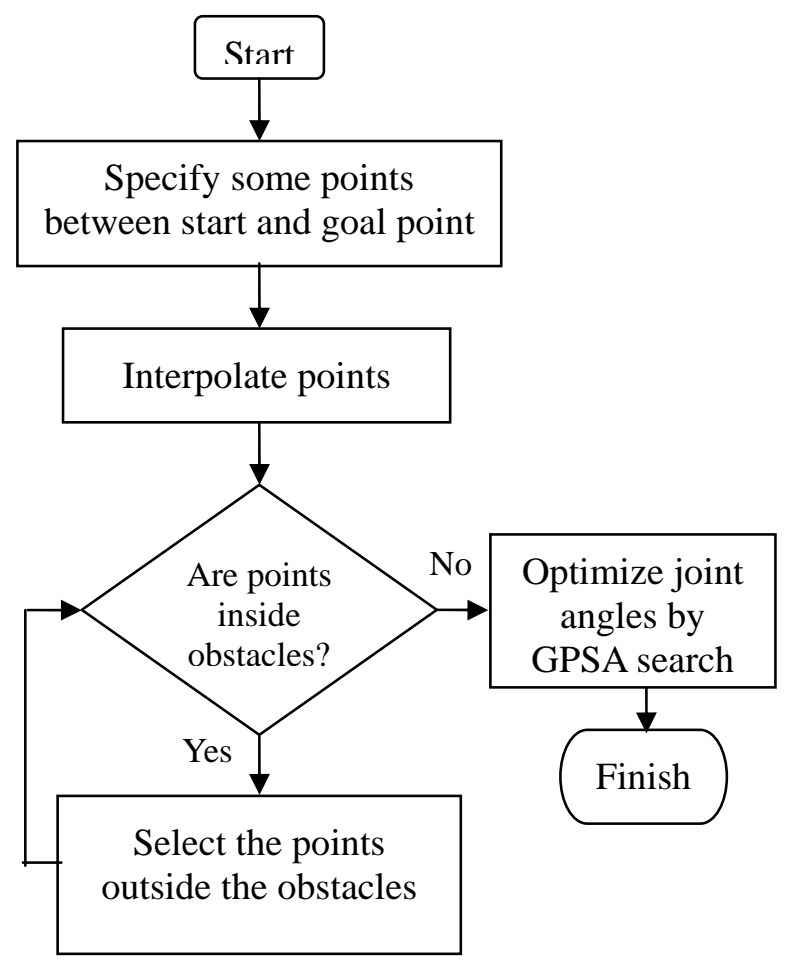

Figure 5: Flowchart of the proposed GPS algorithm.

Specified number of intermediate points is selected and interpolated using cubic-spline interpolation to obtain a smooth trajectory from the starting point to the goal point. In order to avoid manipulator's end-effector collision with surrounding obstacles, interpolated points are checked either inside or outside of obstacles. Points which are inside obstacles will be replaced by new points and checked again to avoid obstacles collision. Consider a circular obstacle for example, if an intermediate point is selected, the algorithm will check whether the point is inside the circle or not by calculating the distance from the center and comparing it with the circle radius. If the point lies inside the circle obstacle, the algorithm will add a tolerance to the calculated radius to make sure that it is outside the circle and evaluates the new coordinates to be used for trajectory interpolation. After ensuring all trajectory points are not trapped inside the obstacles, the Generalized Pattern Search Algorithm finds the optimized joint angles of each link of the manipulator to track the resultant trajectory quite exactly. This algorithm is applied again for the three cases treated before and the results are illustrated in Fig. 6a, 7a, 8a and 6b, 7b, 8b for robot configurations and optimized joint angles respectively. The parameters used in the simulation process are the same for the previous case.

It can be observed from the simulation Fig. 2, 3, 4 and 6, 7, 8 that the end-effector of the redundant manipulator can move freely and does not collide with the workspace obstacles. If all the intermediate points are initially selected outside the obstacles in the course of motion, the algorithm generates a smoother trajectory avoiding obstacles during the time interval.as seen in Fig. 2a, 3a, and 4a. This is due to the excellent tracking performance of the Generalized Pattern Search Algorithm as validated in [1]. 

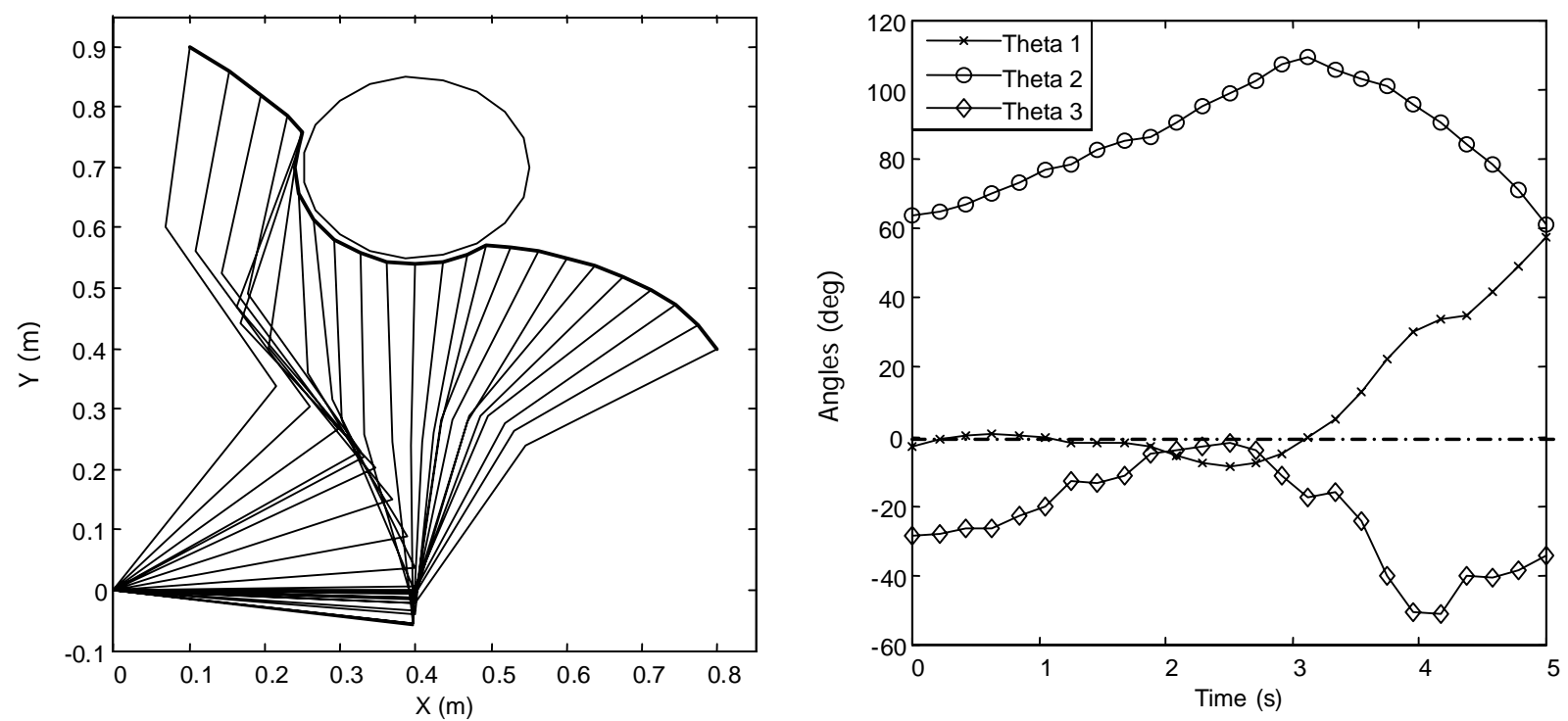

Figure 6a: Robot configuration - one circle obstacle. Figure 6b: Optimized joint angles.
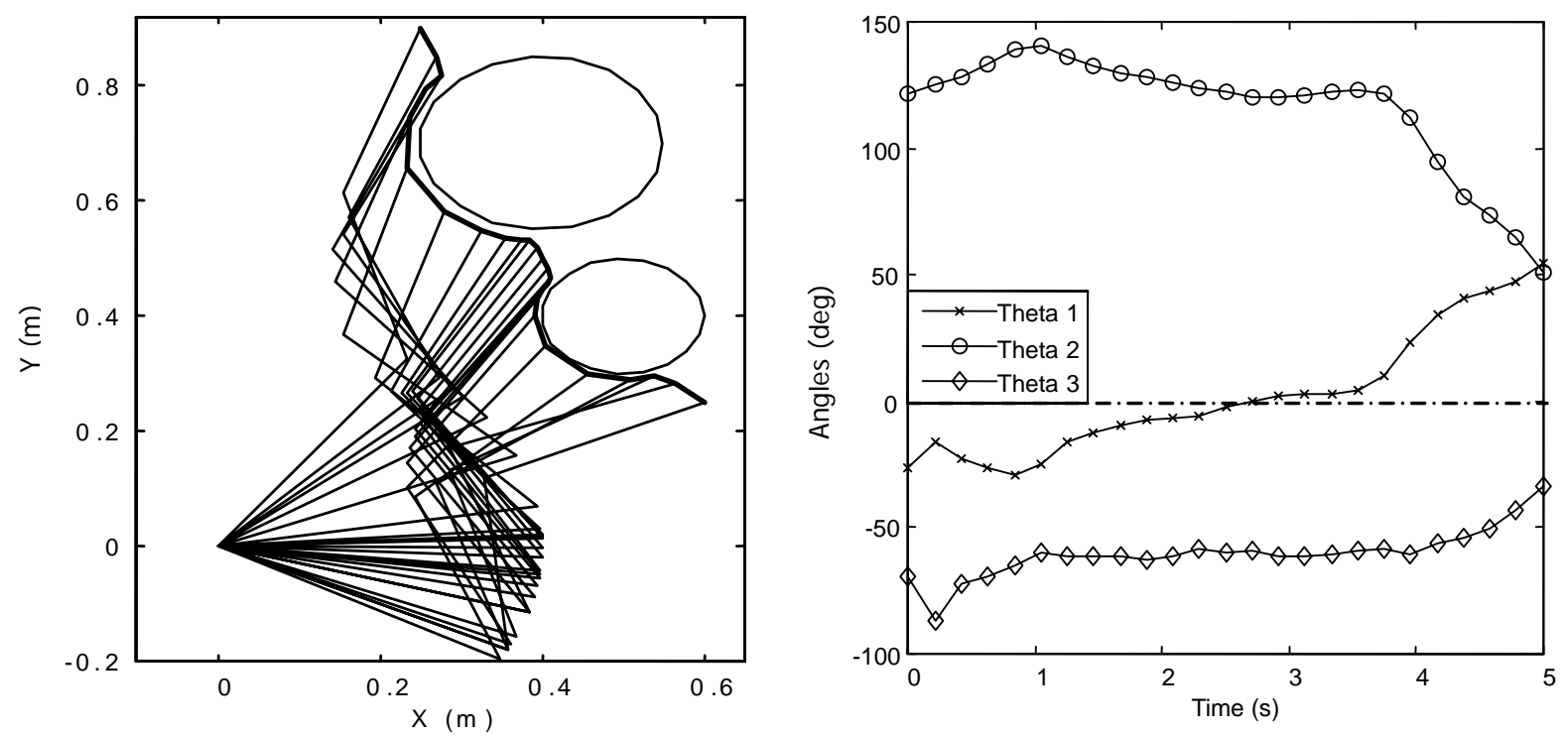

Figure 7a: Robot configuration - two circle obstacle. $\quad$ Figure 7b: Optimized joint angles.
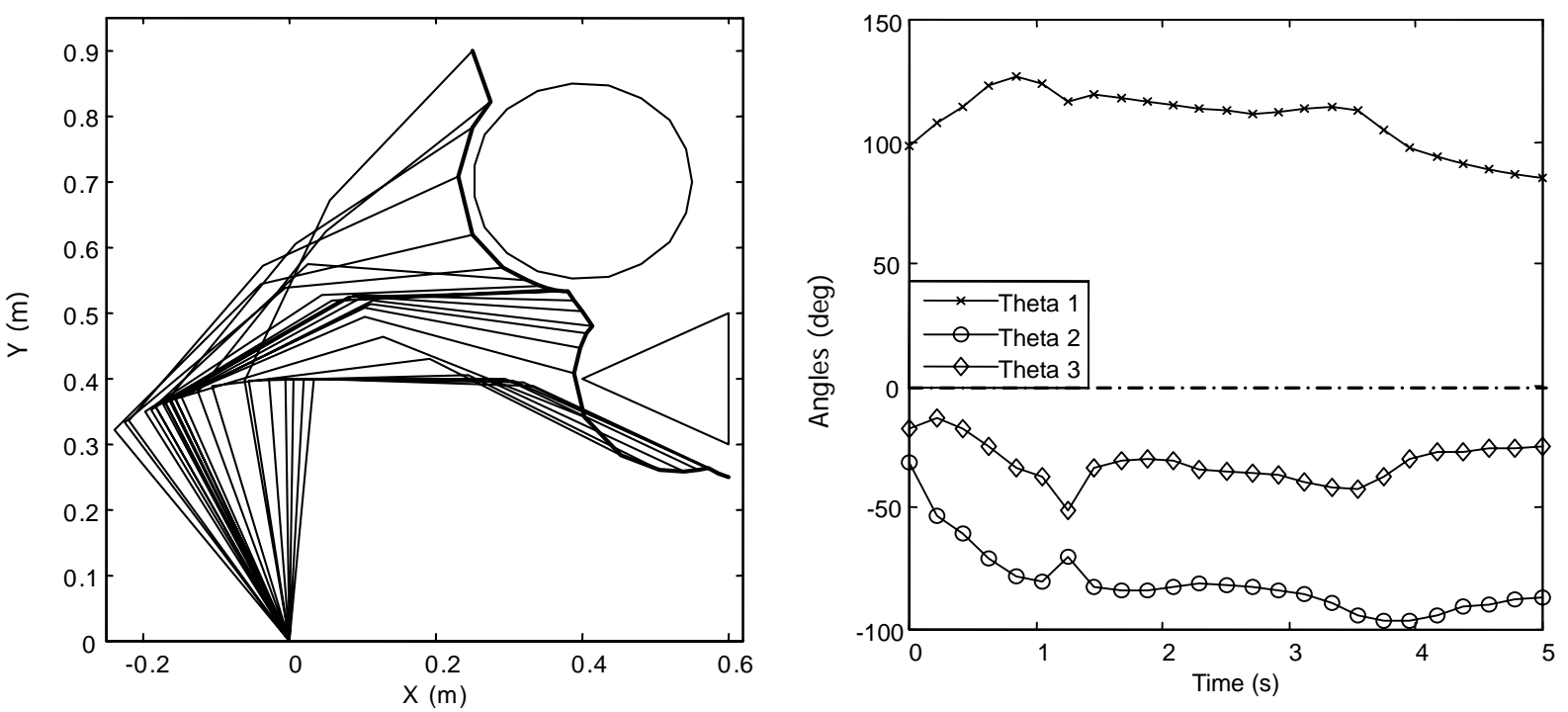

Figure 8a: Robot configuration - circle and triangle. $\quad$ Figure 8b: Optimized joint angles. 
The proposed algorithm is flexible since the selection of the three intermediate points at each stage is arbitrary whether inside or outside the obstacles' workspace and the same cubicspline can be applied for different shapes and geometries of the obstacles.

\section{CONCLUSION}

A Generalized Pattern Search Algorithm combining Genetic Algorithms and Pattern Search is introduced to design a collision-free trajectory for three link planar redundant manipulator. The algorithm starts by assigning three intermediate points between starting and goal points either inside or outside the obstacle workspace and design a cubic-spline trajectory. The algorithm will check if any of the desired trajectory lies inside the obstacles' workspace to replace it with another point outside and repeat the designing process. It is found that if all the three points are selected outside the obstacles' workspace in advance, the resulting trajectory becomes smoother. The introduced algorithm is easy, straight forward and benefits from its excellent tracking property. This algorithm can be also extended to design end-effector trajectory for flexible links manipulator having obstacles in its workspace. Also it can handle dynamic constraints as well as kinematic constraints. Although the Genetic Algorithms are known for slow response, the proposed algorithm can be applied also for the generation of online trajectory planning when the manipulator's workspace has some obstacles in the course of motion.

\section{REFERENCES}

[1] Ata, A. A.; Myo, T. R. (2005). Optimal point-to point trajectory tracking of redundant manipulators using generalized pattern search, International Journal of Advanced Robotic Systems, Vol. 2, No. 3, 239-244

[2] Doyle, A. B.; Jones, D. I. (1996). Robot Path Planning with Genetic Algorithm, The $2^{\text {nd }}$ Portuguese Conference on Automatic Control, 312-318

[3] Goldberg, D. E. (1989). Genetic Algorithm in Search Optimization, and Machine Learning, Addison Wesley

[4] Lee, Y. D.; Lee, B. H. (1997). Genetic trajectory planner for a manipulator with acceleration parameterization, Journal of universal computer Science, Vol. 3

[5] Merchan-Cruz, E. A.; Morris, A. S. (2004). GA based trajectory planner for robot manipulators sharing common workspace, Proceedings of the IASTED International conference on Applied Simulation and Modeling, 96-101

[6] Nearchou, A.; Aspragathos, N. (1994). A collision-detection scheme based on Covex-Hulls concept for generating kinematically feasible robot trajectories, J. Lenarcic and B. Ravani (Editors), Advances in Robot Kinematics and Computational Geometry, Kluwer Academic Publishers, 477-484

[7] Nearchou, A.; Aspragathos, N. (1996). Collision-free continuous path control of manipulators using Genetic Algorithms, Journal of Systems Engineering, Vol. 6, 22-32

[8] Nearchou, A.; Aspragathos, N. (1998). Collision-free continuous trajectory generation using Raster Scanning and Genetic Algorithms, Journal of Intelligent and Robotic Systems, Vol. 16, 351-377

[9] Pack, D., Toussant, G.; Haupt, R. (1996). Robot trajectory planning using a Genetic Algorithms, SPIE, 2824, 171-182

[10] Pires, E. J. S.; Machado, J. A. T.; Oleviera, P. B. M. (2001). An Evolutionary Approach to robot structure and trajectory optimization, ICAR'01-10 $0^{\text {th }}$ International Conference on Advanced Robotics, Budapest, Hungary, 333-338

[11] Rana, A.; Zalzala, A. (1996). An Evolutionary Planner for near time-optimal collision-free motion of multi-arm robotic manipulators, International Conference on Control, 29-35 
[12] Shiller, Z.; Dubowsky, S. (1991). On computing global time-optimal motions of robotic manipulators in the presence of obstacles, IEEE Transactions on Robotics and Automation, Vol. 7, No. 6, 785-797

[13] Tian, L.; Collins, C. (2003). Motion planning for redundant manipulators using a floating point Genetic Algorithms, Journal of Intelligent and Robotic Systems, Theory and Applications, Vol. 38, No. 3-4, 297-312

[14] Tian, L.; Collins, C. (2004). An effective robot trajectory planning method using Genetic Algorithms, Journal of Mechatronics, Vol. 14, 455-470

[15] Wetter, M.; Wright, J. (2003). Comparison of a generalized pattern search and a Genetic Algorithm optimization method, Eighth International IBPSA Conference, Eindhoven, Netherlands, 1401-1408

[16] Yamamoto, M.; Isshiki, Y.; Mohri, A. (1994). Collision-free minimum time trajectory planning for manipulators using Global Search and Gradient Method, Proceedings of IEEE/Rsj/Gi International Conference on Intelligent Robots and Systems, IROS'94, 2184-2191 